\title{
A Corrugated Horn with Broadband Window for W- Band Gyro-devices
}

\author{
Paul McElhinney, Craig R. Donaldson, Liang Zhang, Adrian W. Cross, and Wenlong He \\ Department of Physics, SUPA, University of Strathclyde \\ Glasgow, G4 0NG, Scotland, UK
}

\begin{abstract}
A upgraded W-band corrugated horn including a broadband vacuum window for use in gyro-devices has been designed, constructed and experimentally measured. The horn and multi-layer vacuum window converts a cylindrical $T_{11}$ mode into the free space quasi-optical TEMov mode over the frequency band of $90-100 \mathrm{GHz}$ with a reflection better than -30 $\mathrm{dB}$ and a coupling efficiency of $\sim 99.4 \%$.
\end{abstract}

Keywords-corrugated horn; gyro-TWA; gyro-BWO; quasioptical; broadband window

\section{CORRUGATED LAUNCHER AND WINDOW}

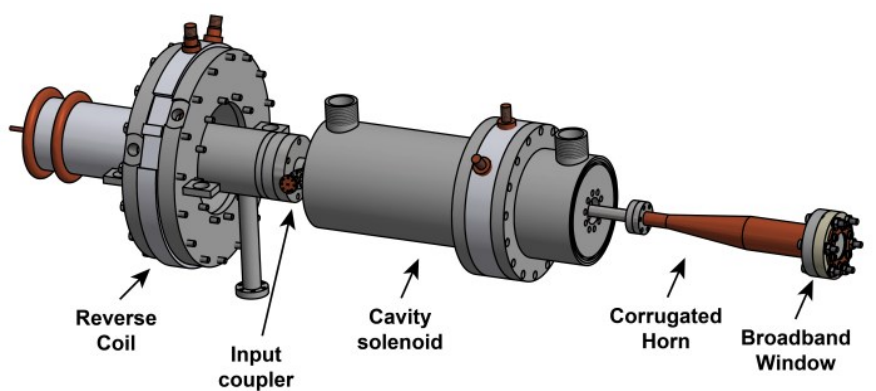

Fig. 1. CAD system drawing, showing horn in situ.

A W-band gyrotron traveling wave amplifier (gyro-TWA) and gyrotron backward wave oscillator (gyro-BWO) [1] based on a cusp electron beam source [2-4] and a helically corrugated interaction region (HCIR) [5] have been developed to provide a continuously tunable and continuous wave $(\mathrm{CW})$ source with a power output of $\sim 5 \mathrm{~kW}$ and $\sim 10 \mathrm{~kW}$ respectively. The gyro-TWA is designed to have a $3 \mathrm{~dB}$ frequency bandwidth of $90-100 \mathrm{GHz}$ while the gyro-BWO demonstrated a tuning range of $88-102.5 \mathrm{GHz}$ with an output power of $12 \mathrm{~kW} \mathrm{[6].} \mathrm{Both} \mathrm{the} \mathrm{gyro-TWA} \mathrm{and} \mathrm{BWO} \mathrm{operate}$ using an elliptically polarized $\mathrm{TE}_{11}$ mode, but to provide a source that is suitable for applications an output that can convert the $\mathrm{TE}_{11}$ to a quasi-optical mode is desirable. This can be achieved using a corrugated mode converting horn [7], which transforms the fundamental $\mathrm{TE}_{11}$ mode within the gyroTWA to a hybrid mode that is closely coupled to the fundamental free space Gaussian mode $\left(\mathrm{TEM}_{00}\right)$ [8]. The radiated Gaussian beam may then pass through the vacuum window [9] unperturbed with a very low reflection.

The mode converting horn is seen in the context of the entire system in a CAD representation (fig. 1) and in the system schematic (fig. 2), which demonstrates the configuration of the system in terms of its primary components and processes.

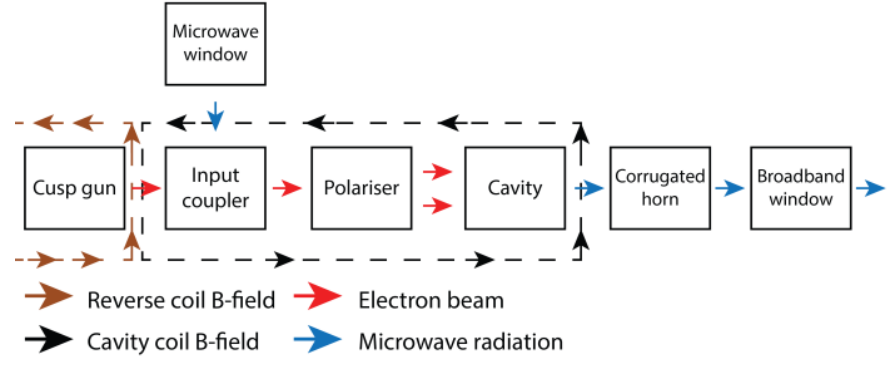

Fig. 2. Conceptual schematic of the Gyro-TWA system

This type of output coupler is advantageous due to the greater bandwidth and the capability to operate with a source that is continuously tunable. The reduction of unwanted reflections was the primary consideration in the design and optimization process, which was carried out using a mode matching method and numerically validated by comparing the aperture fields with the fundamental Gaussian mode. The optimal reflection was best achieved using a $\sin ^{2}$ profile [10] in conjunction with a multi-layer vacuum window; however, the coupling efficiency can be increased to greater than $99.8 \%$ by introducing higher order, $\mathrm{HE}_{1 \mathrm{n}}$ modes to the aperture field. This can be achieved by introducing a phase matching straight corrugated waveguide section in addition to the initial profiled horn section, which provides a means to combine the $\mathrm{HE}_{11}$ and $\mathrm{HE}_{12}$ waveguide modes, generated by the profiled horn, with the correct amplitude and phase at the aperture.

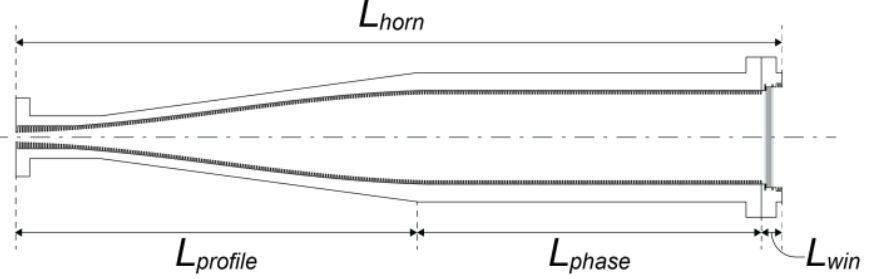

Fig. 3. Side elevation showing $\sin ^{2}$ profiled section, phase matching section and window.

Consideration must also be made for the window, which is an integral part of the horn and is located at the aperture side of the straight waveguide section, with the electron beam dump before the input to the horn. The window is based on a multi-layer window that was previously implemented as part of our gyro-TWA/BWO design [11].

The placement of this window at the horn aperture, after the horn, simplifies the design of the window structure by placing it after the conversion to the hybrid mode where nearly all the power is located in the center of the waveguide 
and away from the walls. This enables a window design that is radially large compared with the beam waist and also allows larger steps in the radius of the ceramic and quartz discs without an appreciable effect on the radiation. This method greatly simplifies the mechanical construction and vacuum brazing. A schematic of the improved horn with the $\sin ^{2}$ profiled section, the phase matching section and the attached window is shown in fig. 3 .

\section{EXPERIMENTAL RESULTS}

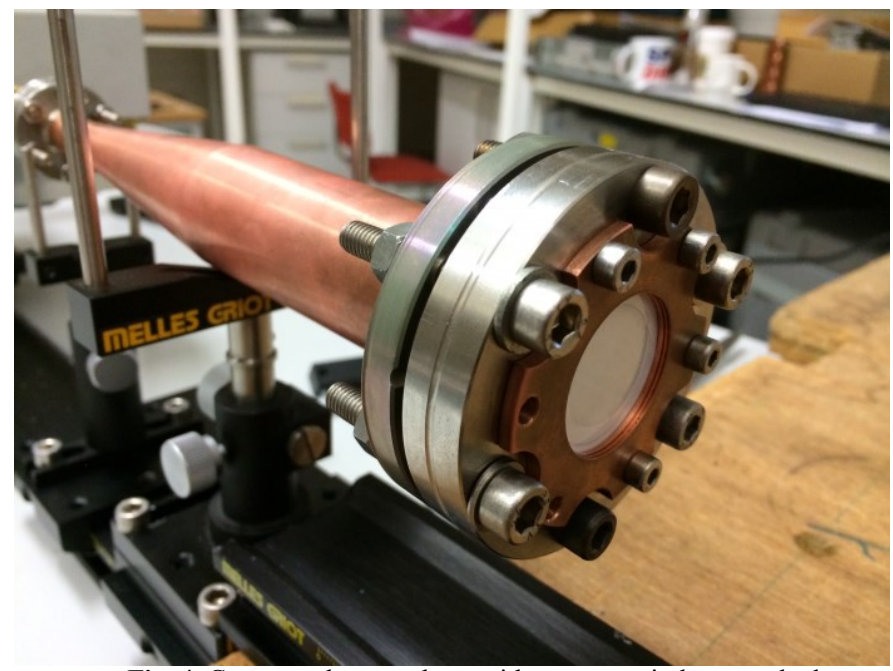

Fig. 4. Corrugated copper horn with vacuum window attached.

This horn was constructed by the electroforming copper onto an aluminum substrate and the completed millimeter wave component was tested on a W-band Anritsu 3738A VNA. The reflection from the horn was determined by one port measurement where microwaves were radiated into free space and the far-field and near-field aperture measurements were also carried out in this way using a WR-10 receiving horn on the second port. Fig. 4 shows the corrugated horn and window attached to the VNA and far-field rig.

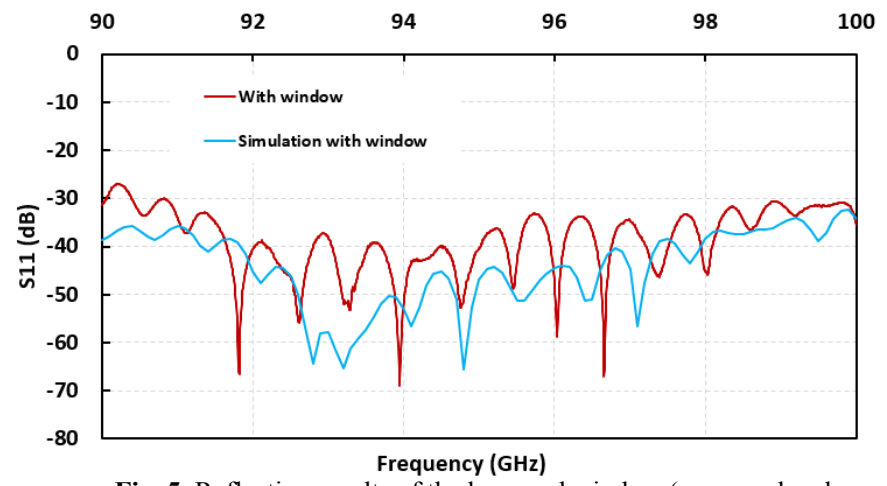

Fig. 5. Reflection results of the horn and window (measured and simulated).

The measurement of the reflection $\left(\mathrm{S}_{11}\right)$ from the horn was carried out with the window in place after the window assembly was vacuum tested and sealed. Sealing the vacuum by tightening the bolts that connect the window and horn can cause small changes in the gap spacing between the dielectric window layers, which dramatically changes the performance of the window. The measured performance (fig. 5) is very close to the simulations and demonstrates that the optimum window spacing has been achieved for the lowest reflection.
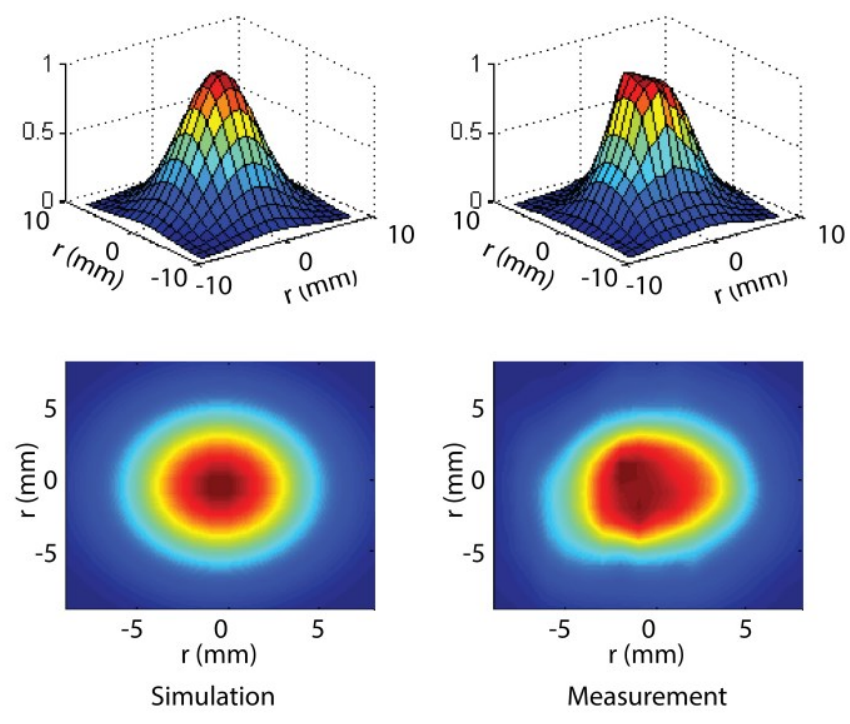

Fig. 6. Near-field contour plot (theory and measured).

From far-field measurements, it is evident that the $-30 \mathrm{~dB}$ edge was within a half angle of 14.5 degrees and the pattern showed more than $99 \%$ of the output power was within 29 degrees. The simulated results demonstrated that the horn is coupled to the fundamental Gaussian with an efficiency of $\sim 99.4 \%$. A near-field scan was carried out to confirm these results and the data are shown as a contour plot in fig. 6 .

\section{SUMMARY}

A upgraded mode converting horn and window system has been developed to produce a Gaussian output with a reflection over the $90-100 \mathrm{GHz}$ bandwidth of better than $-30 \mathrm{~dB}$. This second generation horn includes an output window, which allows for better integration with the gyro-system and has a coupling between the $\mathrm{HE}_{11}$ mode and the free space Gaussian mode of $99.4 \%$ with the window.

\section{References}

[1] W. He, C. R. Donaldson, F. Li, L. Zhang, A. W. Cross, A. D. R. Phelps, K. Ronald, C. W. Robertson, C. G. Whyte, and A. R. Young, "W-band gyrodevices using helically corrugated waveguide and cusp gun: Design, simulation and experiment," TST, 4, pp. 9-19, 2011.

[2] W. He, C. G. Whyte, E. G. Rafferty, A. W. Cross, A. D. R. Phelps, K. Ronald, A. R. Young, C. W. Robertson, D. C. Speirs, and D. H. Rowlands, "Axis-encircling electron beam generation using a smooth magnetic cusp for gyrodevices," Appl. Phys. Lett. 93, 121501, 2008

[3] C. R. Donaldson, W. He, A. W. Cross, A. D. R. Phelps, F. Li, K. Ronald, C. W. Robertson, C. G Whyte, A. R. Young, L. Zhang, "Design and numerical optimization of a cusp-based electron beam for millimetre-wave gyro-devices," IEEE Trans. Plasma Sci., 37, pp. 2153-2157, 2009.

[4] C. R. Donaldson, W. He, A. W. Cross, F. Li, A. D. R. Phelps, L. Zhang, K. Ronald, C. W. Robertson, C. G. Whyte, A. R. Young, "A cusp electron gun for millimeter wave gyro-devices”, Appl. Phys. Lett., 96, 141501, 2010.

[5] L. Zhang, W. He, K. Ronald, A. D. R. Phelps, C. G. Whyte, C. W. Robertson, A. R. Young, C. R. Donaldson, and A. W. Cross, "Multi-mode 
coupling wave theory for helically corrugated waveguide," IEEE Trans. Microw. Theory Tech., 60, pp. 1-7, 2012

[6] W. He, C. R. Donaldson, L. Zhang, K. Ronald, P. McElhinney, A. W. Cross, "High power wideband gyrotron backward wave oscillator operating towards the terahertz region," Phys. Rev. Lett. 110, 165101, 2013.

[7] J. E. McKay, D. A. Robertson, P. A. S. Cruickshank, R. I.. Hunter, D. R. Bolton, R. J. Wylde, G.M. Smith, IEEE Trans. on Antennas and Propagation., 61, pp. 1714-1721, 2013.

[8] P. McElhinney, C. R. Donaldson, L. Zhang, and W. He, "A high directivity broadband corrugated horn for W-band gyro-devices," IEEE Trans. Antennas Propag. 61, pp. 1453-1456, 2013.
[9] C. R. Donaldson, W. He, L. Zhang, and A.W. Cross, "A W-band multilayer microwave window for pulsed operation of gyro-devices," IEEE Microw. Wireless Compon. Lett., 23, pp. 237-239, 2013.

[10] P. J. B. Clarricoats and A. D. Olver, Corrugated Horns for Microwave Antennas. Peregrinus, London, UK, 1984.

[11] C. R. Donaldson, W. He, L. Zhang, and A. W. Cross, "A W-band multilayer microwave window for pulsed operation of gyro-devices," IEEE Microw. Wireless Compon. Lett., 23, pp. 237-239, 2013. 